\title{
Maximal charge injection of a uniform separated electron pulse train in a drift space
}

\author{
Y. L. Liu, ${ }^{1,3}$ P. Zhang, ${ }^{2}$ S. H. Chen, ${ }^{1}$ and L. K. Ang ${ }^{3, *}$ \\ ${ }^{1}$ Department of Physics, National Central University, Jhongli 32001, Taiwan \\ ${ }^{2}$ Department of Nuclear Engineering and Radiological Sciences, \\ University of Michigan, Ann Arbor, Michigan 48109-2104, USA \\ ${ }^{3}$ Engineering Product Development, Singapore University of Technology and Design, Singapore 487372
}

(Received 3 September 2015; published 21 December 2015)

\begin{abstract}
A charge sheet model is proposed to study the space charge effect and uniformity of charge separation of an electron pulse train in a drift space. An analytical formula is derived for the charge density limit as a function of gap spacing, injecting energy and pulse separation. To consider the relativistic effects, the theoretical results are verified by numerical solutions up to $80 \mathrm{MeV}$. This model can be applied to the design of Smith-Purcell radiation, multiple-pulse electron beam for time resolved electron microscopy, and to free electron laser.
\end{abstract}

DOI: 10.1103/PhysRevSTAB.18.123402

PACS numbers: $52.59 . \mathrm{Sa}$

\section{INTRODUCTION}

Consecutive electron pulses or multipulse comb beams, which are generated by comb-like laser pulse illuminated photocathode [1] or direct laser electron accelerator (DLA) [2], have wide applications, such as terahertz radiation sources [3], free electron laser (FEL) [4], high harmonics generation (HHG) processes [5] and four-dimensional ultrafast electron microscopy (UEM) [6]. The pre-bunched charge sources have the potential to greatly enhance the efficiency or power of the devices, currently attracting the attention of many scientists.

Current density and pulse duration of the electron pulses play an important role to these applications. Take Ref. [3] as an example, a scheme was proposed to enhance SmithPurcell (SP) radiation [7] in the terahertz wavelength range by generating a train of prebunched electron beams. In this scheme, sufficient charge number per pulse is required to have enhanced SP radiation [8]. However, the space charge effect at high charge density may destroy the temporal profile of the pulses. Hence, studying the influence of space charge effect of multiple electron pulses in drift space is important.

At high current regime, the space charge effect will limit the maximum injected current density which is the socalled the space charge limited current (SCLC) density. Considering a one-dimensional (1D) planar accelerating diode with gap distance $L$ and gap voltage $V_{g}$, the maximal steady state SCLC density is given by the classical ChildLangmuir (CL) law $[9,10]$ given by

\footnotetext{
*ricky_ang@sutd.edu.sg
}

Published by the American Physical Society under the terms of the Creative Commons Attribution 3.0 License. Further distribution of this work must maintain attribution to the author(s) and the published article's title, journal citation, and DOI.

$$
J_{\mathrm{CL}}=\frac{4}{9} \epsilon_{0} \sqrt{\frac{2 e}{m}} \frac{V_{g}^{\frac{3}{2}}}{L^{2}},
$$

where $e$ and $m$ are, respectively, the charge and mass of the electron, and $\epsilon_{0}$ is the free space permittivity (note that this equation presumes an initial velocity of zero). After leaving the diode's accelerating region, the electrons enter the drift space region with a velocity $\sqrt{2 e V_{g} / m}$ [a finite initial velocity from the injecting surface is considered, which is different from Eq. (1)]. For a drift space of length $d$ and of zero electric field, the SCLC density in a drift space [11] is expressed as

$$
J_{\mathrm{DSCL}}=\frac{32}{9} \epsilon_{0} \sqrt{\frac{2 e}{m}} \frac{V_{g}^{\frac{3}{2}}}{d^{2}} .
$$

In past years, the studies of SCLC in a diode and a drift space have been revised extensively to consider various effects such as finite emission area [12-16], short pulse length and relativistic (and quantum) effects [17-24], semianalytical scaling for cylindrical and spherical diodes $[25,26]$.

In this paper, we extend our previous work [24] of calculating the maximum charge density for a uniform electron pulse train in a diode to a drift space. In Ref. [24], a 1D model to study the space charge limited charge injection of a train of multiple electron pulse into a diode were presented. The charge sheet model was used to obtain an analytical formula, which can quickly provide such upper limit of charge density injection once the values of gap spacing, gap voltage, and the initial time separation between the pulses are provided.

In contrast to an accelerating diode, the boundary conditions at upstream and downstream ends of a drift space are of the same potential, and the electrons injected 
from the upstream end with an initial velocity that is determined by the accelerating diode. Unlike a diode for which the SCLC is determined by the zero electric field at the injecting end (or the cathode), the criteria of SCLC for a drift space is the minimal electric field occurring at the midpoint. By constructing a 1D charge sheet model, an analytical or semianalytical formula was derived to estimate the upper limit of the charge density per pulse for any given pulse interval, gap spacing, and injecting velocity in a drift space. To verify the formula, the consecutive electron pulses in Ref. [3] are used as an example to determine the maximum charge density per pulse while the pulse interval almost remains constant. By including relativistic effect, the model developed in the study is still valid for devices with the electron beam energy up to $80 \mathrm{MeV}$ [2].

\section{ANALYTICAL MODEL}

Consider a single charge sheet of charge density $\sigma$ injected into a drift space with an initial velocity $v_{0}=$ $\sqrt{2 e V_{g} / m}$ (injected by an accelerating diode with gap voltage $V_{g}$ ), as shown in Fig. 1(a). By solving Poisson's equation $\varphi^{\prime \prime}=-\frac{\sigma}{\epsilon_{0}} \delta\left(x-x_{1}\right)$ with the grounded boundary conditions on both electrodes $[\varphi(0)=\varphi(d)=0]$, the electric field profiles are $E_{1}=\frac{\sigma}{\epsilon_{0}}\left(\frac{x_{1}}{d}\right)$ and $E_{2}=$ $-\frac{\sigma}{\epsilon_{0}}\left(1-\frac{x_{1}}{d}\right)$, where $E_{1}$ and $E_{2}$ are the fields acting on the downstream electrode or anode $(x=d)$ and on the upstream electrode or cathode $(x=0)$, respectively. The corresponding potential profiles of both regions as shown

(a)

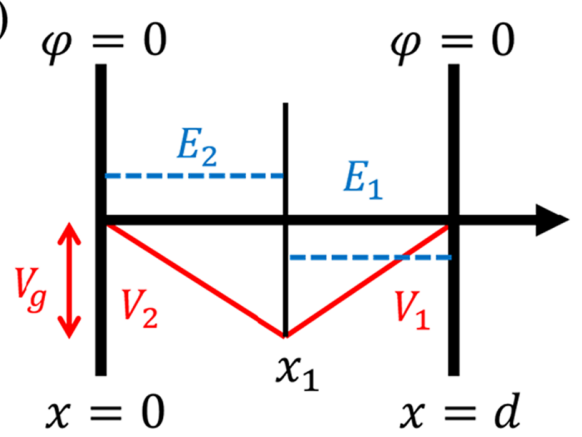

(b)

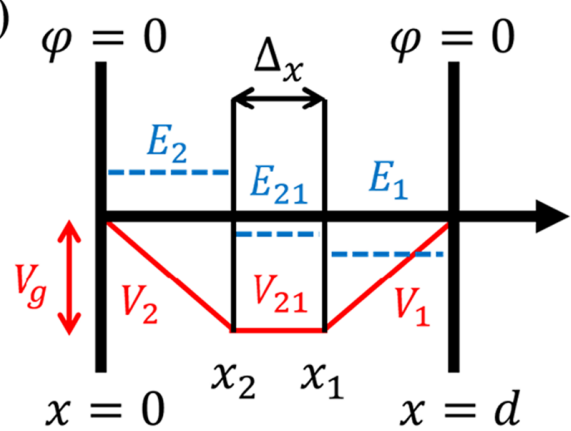

FIG. 1. A drift space with gap spacing $d$ with (a) single pulse injection and (b) two pulse injections, respectively. in Fig. 1(a) are respectively $V_{1}(x)=-E_{1} x+E_{1} d$ and $V_{2}(x)=E_{2} x[11]$.

If the charge sheet with the maximum density is located at the center of the drift space $(x=d / 2)$, the potential field profile is symmetric with respect to the mid-point, and the potential minimum at the center of the drift space equals to $V_{g}$ based on energy conservation, i.e., $V_{1,2}\left(\frac{d}{2}\right)=$ $-V_{g}=\frac{d}{2} E_{1}$. Thus we have the maximum charge density $\sigma_{1}=-4 \epsilon_{0} \frac{V_{g}}{d}$ and the SCLC density is given by $J_{1}=\frac{\sigma_{1}}{\tau_{p}}$, where $\tau_{p}$ is the pulse duration [22]. A normalized time scale $X_{\mathrm{DSCL}} \equiv \frac{\tau_{p}}{T_{\mathrm{DSCL}}}$ is introduced to define the ratio between the pulse duration and the transit time $T_{\mathrm{DSCL}}$ of SCLC in a drift space. The transit time is expressed as $T_{\mathrm{DSCL}}=\frac{3 d}{\sqrt{8 e V_{g} / m}}$ in the classical regime [11], and $T_{\mathrm{RDSCL}}=\frac{2 d}{c} \frac{\left(\sqrt{\gamma_{0}^{2}-1}-\sqrt{\gamma_{m}^{2}-1}\right)^{1 / 2}}{G\left(\gamma_{0}\right)}$ in the relativistic regime. Here, $G\left(\gamma_{0}\right)=$ $\int_{\gamma_{m}}^{\gamma_{0}}\left(\sqrt{r^{2}-1}-\sqrt{\gamma_{m}^{2}-1}\right)^{-1 / 2} d r, \gamma_{0}$ and $\gamma_{m}$ are the maximum and minimum Lorentz factors, respectively.

By solving $\varphi^{\prime \prime}=-\frac{\sigma}{\epsilon_{0}} \sum_{n=1}^{N} \delta\left(x-x_{n}\right)$ for $N$ number of pulses and assuming that pulses are distributed symmetrically with equal pulse spacing $\Delta_{x}$, the potential minimum in the center of the drift space is

$$
-V_{g}= \begin{cases}\frac{d}{2} \frac{\sigma}{\epsilon_{0}}\left[\sum_{n=1}^{\frac{N+1}{2}}\left(\frac{1}{2}-(n-1) \frac{\Delta x}{d}\right)-\frac{1}{2}\right], & N=\text { odd } \\ \frac{d}{2} \frac{\sigma}{\epsilon_{0}} \sum_{n=1}^{\frac{N}{2}}\left(1-(2 n-1) \frac{\Delta x}{d}\right), & N=\text { even }\end{cases}
$$

Letting $\Delta_{x}=v_{0} \Delta_{t}$, the maximum charge density is given by

$$
\frac{\sigma_{N}}{\sigma_{1}}= \begin{cases}{\left[N-\frac{v_{0} \Delta_{t}}{d}\left(\frac{N^{2}-1}{2}\right)\right]^{-1},} & N=\text { odd } \\ {\left[N-\frac{v_{0} \Delta_{t}}{d}\left(\frac{N^{2}}{2}\right)\right]^{-1},} & N=\text { even }\end{cases}
$$

For better illustrations, a simple diagram for two charge sheets is shown in Fig. 1(b). Note that the two expressions in Eq. (4) are identical at $N \gg 1$, and $\sigma_{N}=\frac{\sigma_{1}}{N}$ recovers to the result of single charge sheet at $v_{0} \Delta_{t} \rightarrow 0$. The maximum number of charge sheets in the drift space can be estimated by $N_{\max } \cong\left\lceil\frac{T_{0}}{\Delta_{t}}\right\rceil$, where $T_{0} \cong \frac{d}{v_{0}}$ is the transit time of single charge sheet.

To consider the dynamic behavior of the charge sheets, we solve the normalized equation of motion (EOM) numerically for the position $x_{n}(\mathrm{t})$ of each sheet, which is injected into the drift space. The normalized charge density (in terms of $\sigma_{1}$ ) is

$$
\bar{\sigma}(\bar{t})=\frac{\sigma_{N}}{\sigma_{1}} \sum_{n=1}^{N} \bar{x}_{n}(\bar{t}),
$$


where $\bar{x}_{n}=\frac{x_{n}(t)}{d}$ is the normalized position of $n$th sheet at normalized time $\bar{t}=\frac{t}{T_{\mathrm{DSCL}}}$, respectively. The normalized temporal electric field (in terms of $\frac{\sigma_{1}}{\epsilon_{0}}$ ) acting on each charge sheet is

$$
\bar{E}_{n}(\bar{t})=\frac{\sigma_{N}}{\sigma_{1}} \sum_{n=1}^{N} \bar{x}_{n}(\bar{t})-\frac{\sigma_{N}}{\sigma_{1}}\left(n-\frac{1}{2}\right) .
$$

The normalized EOM is given by

$$
\left\{\begin{array}{l}
\frac{d \bar{v}_{n}}{d \bar{t}}=0.75 \bar{E}_{n}(\bar{t}) \\
\frac{d \bar{x}_{n}}{d t}=1.5 \bar{v}_{n}(\bar{t})
\end{array}\right.
$$

which are solved numerically with initial conditions: $\bar{x}_{n}(0)=0 ; \quad \bar{v}_{n}(0)=1, \quad$ where $\quad \bar{v}_{n}=v_{n} / v_{0} . \quad$ Using $J(t)=\frac{d \sigma(t)}{d t}$, we derive the normalized current density (in terms of $\left.\frac{\sigma_{1}}{T_{\mathrm{DSCL}}}\right)$ as

$$
\bar{J}(\bar{t})=1.5\left(\frac{\sigma_{N}}{\sigma_{1}}\right) \sum_{n=1}^{N} \bar{v}_{n}(\bar{t}) .
$$

\section{RESULTS}

As an example, we consider a $50 \mathrm{keV}$ prebunched electron beam with temporal pulse spacing $\Delta_{t}=2.16 \mathrm{ps}$ passing above a $5 \mathrm{~cm}$ grating [3]. The corresponding transit time for single charge sheet is $T_{0} \cong 377 \mathrm{ps}$ and the maximum number of charge sheet is $N_{\max } \cong\left\lceil\frac{T_{0}}{\Delta_{t}}\right\rceil=175$.

In Figs 2(a) and 2(d), we show the trajectories of each charge sheet and its corresponding current density $(J)$ by solving Eqs. (7) and (8) for a given charge density $\sigma=\sigma_{175}$ from Eq. (4), respectively. It is found that some of the injected charge sheets are reflected due to the space charge effect and the negative current density is observed as shown in Fig. 2(d). This finding implies that Eq. (4) has overestimated the maximal charge density. Hence, a lower charge density is expected to reduce the space charge effect in order to maintain a temporally uniform pulse structure. In doing so, we introduce $f(<1)$ as a fraction of the maximum charge density $\sigma_{N}$ [from Eq. (4)], which gives $\sigma=f \sigma_{N}$. Figure 2(b) and 2(e) show the trajectories of each charge sheet and the current density with a given charge density $\sigma=0.7 \sigma_{175}$ for $f=0.7$. By defining $\Delta_{f}$ to be the final temporal separation of the last two charge sheets arriving at the anode, we can use $\Delta_{f}$ to measure the uniformity of pulse intervals. From Fig. 2(b), there is expansion of charge sheet separation as shown in Fig. 2(g) [magnification of Fig. 2(b) near $x=1$ region, and $\left.\Delta_{f}=6.35 \mathrm{ps}\right]$. This suggests that the charge density is still too large even if there are no reflections of charge sheets. By reducing to $f=0.1$, Figs. 2(c) and 2(f) show the trajectories of all charge sheets and the current density at
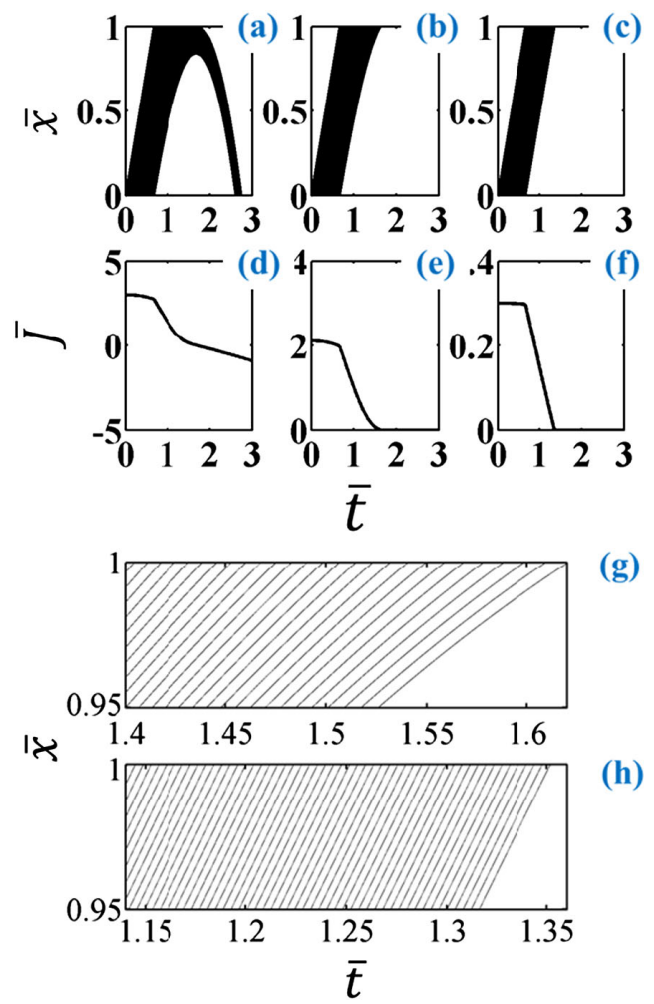

(g)

FIG. 2. The trajectories of each beam [(a), (b) and (c)] and current density [(d), (e) and (f)] in a drift space with gap spacing $d=5[\mathrm{~cm}]$ and injected kinetic energy $50[\mathrm{keV}]$ for $N=30$ and $\Delta_{t}=2.16[\mathrm{ps}]$ at $\sigma=\sigma_{N=175}\left[\right.$ (a) and (d)], $\sigma=0.7 \sigma_{N=175}[$ (b) and (e) $]$ and $\sigma=0.1 \sigma_{N=175}$ [(c) and (f)]. The last 2 figures: (g) and (h) are the magnification of (b) and (c), respectively in region near to $x=1$. In the figure, the normalized parameters are defined as $\bar{x} \equiv \frac{x}{d}, \bar{t} \equiv \frac{t}{T_{\mathrm{DSCL}}}$ and $\bar{J} \equiv \frac{\mathrm{J}}{\sigma_{1} / T_{\mathrm{DSCL}}}$.

$\sigma=0.1 \sigma_{175}$. In these two figures, a temporally uniform charge sheet train is demonstrated in Fig. 2(h) [magnification of Fig. 2(c) near $x=1$ region, and $\Delta_{f}=2.26 \mathrm{ps]}$, and the current density maintains a constant until the last charge sheet passing though the gap.

The open circles in Fig. 3 show the final time intervals $\Delta_{f}$ as a function of $f$ for $N=19$ (red), 175 (black) and 320 (blue), which indicates that $\Delta_{f}$ converges to $\Delta_{t}$ at $f \rightarrow 0.1$. For larger $N$, the deviation of $\Delta_{f}$ from $\Delta_{t}$ occurs at smaller $f$ as expected. From the experiment reported in Ref. [3], the number of pulse used was reported to be $N=19$ and $N=320$. From our results, the differences between $\Delta_{f}$ and $\Delta_{t}$ are within $10 \%$ at $f \rightarrow 0.1$ and is further reduced to $2 \%$ at $f \rightarrow 0.01$. Thus our model can be used to correctly estimate the maximal charge density based on the acceptable nonuniformity of the pulse train in various applications, including terahertz radiation sources [3], free electron laser (FEL) [4], high harmonics generation (HHG) processes [5], and four-dimensional ultrafast electron microscopy (UEM) [6], etc. Dependent on specific application, if the proposed $10 \%$ deviation is not good enough, the model 


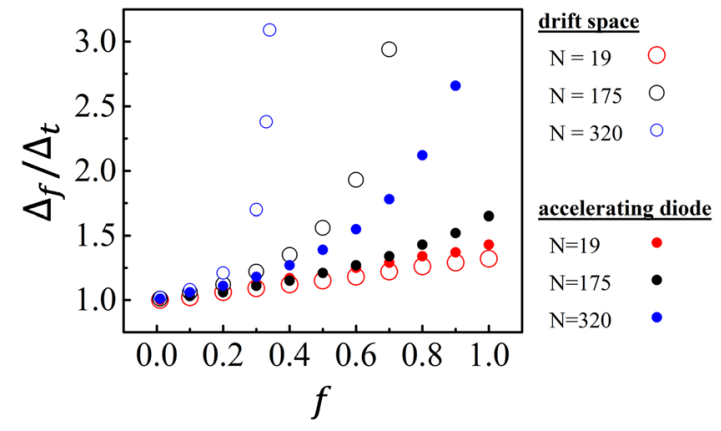

FIG. 3. The dependence of $\Delta_{f}$ (in terms of $\Delta_{t}$ ) as a function of $f$ for $\Delta_{t}=2.16[\mathrm{ps}]$ for $N=19$ (red), 175 (black) and 320 (blue). The open circles are for drift space, and the solid circles are for accelerating diode.

can be revised to calculate smaller value of $f$ in order to have smaller deviation.

To compare with the diode model [24], the same gap spacing $d=5 \mathrm{~cm}$, gap voltage $V_{g}=50 \mathrm{kV}$, and the temporal pulse spacing $\Delta_{t}=2.16 \mathrm{ps}$ are used in the calculation. According to our previous work, the maximum number of charge sheets in a diode is $N_{\max }=350$ and the corresponding maximum charge density is $\sigma_{N}=\frac{-\epsilon_{0} V_{g} / d}{N}\left[1-\frac{1}{2}\left(\frac{q V_{g}}{m d}\right) \frac{\Delta_{t}^{2}}{d}\left(\frac{N-1}{2}\right)\right]^{-1}$. The solid circles (for the diode model) in Fig. 3 show the final time intervals $\Delta_{f}$ as a function of $f$ for $N=19$ (red), 175 (black) and 320 (blue), which shows that $\Delta_{f}$ also converges to $\Delta_{t}$ at $f \rightarrow 0.1$. The comparison implies that drift space cases vary more significantly to $f$ when the number of pulse is increased from 19 to 350 . It is interesting to note the ratio $\Delta_{f} / \Delta_{t}$ is higher at small $N$ values for the drift space as compared to the accelerating diode. At higher values of $N$, the ratio $\Delta_{f} / \Delta_{t}$ will be higher for the accelerating diode. For small $N=19$ case in the accelerating diode, there is an external field in the diode which is larger than the electric fields between the 2 charge sheets. There is no external applied fields in the drift space, hence, only the interacting electric fields will affect the motions of charge sheets. We suspect that at small $N$ case, the space charge effect between the charge sheets is less dominant for which the external field is more important (like accelerating diode). This may explain why the ratio for $N=19$ for drift space is less than accelerating diode. At larger values of $N$, the interacting between the charge sheets will become more important as compared to the external field, which may explain why the ratio at $N=175$ for drift space becomes larger as compared to the accelerating diode case. The transition between this 2 competing effects should be around $N=19$ as the difference is small at this regime of smaller $N$.

The classical model of EOM presented above can be extended to include the relativistic effect when the injected kinetic energy is comparable or larger than $0.511 \mathrm{MeV}$. As an example, the recent DLA [2] enables the generation of an electron bunch train at $80 \mathrm{MeV}$. In the relativistic regime, Eq. (7) becomes

$$
\left\{\begin{array}{l}
\frac{d \bar{u}_{n}}{d \bar{t}} 8\left(\gamma_{0}-1\right) \frac{\left(\sqrt{\gamma_{0}^{2}-1}-\sqrt{\gamma_{m}^{2}-1}\right)^{1 / 2}}{G\left(\gamma_{0}\right)}\left[\bar{E}_{n} \sum_{n=2}^{N} \bar{x}_{n}(\bar{t})-\bar{E}_{n}\left(n-\frac{1}{2}\right)\right], \\
\frac{d \bar{x}_{n}}{d t}=2 \frac{\left(\sqrt{\gamma_{0}^{2}-1}-\sqrt{\gamma_{m}^{2}-1}\right)^{1 / 2}}{G\left(\gamma_{0}\right)} \bar{u}_{n}(\bar{t})
\end{array}\right.
$$

where $\bar{u}_{n}=u_{n} / c$ is the normalized momentum with respect to the speed of light $c$, and the corresponding normalized time $\bar{t}=\frac{t}{T_{\mathrm{RDSCL}}}$. According to DLA simulations [2], we choose the gap spacing $d=5[\mathrm{~mm}]$, time interval $\Delta_{t}=3[\mathrm{fs}]$, the relativistic injected kinetic energy $K=$ $80[\mathrm{MeV}]$ and $N_{\max }=36$ to verify whether the criteria $f=$ 0.1 for the convergence $\Delta_{f}$ to $\Delta_{t}$ remains valid. In this setting, there are about 10 microbunches in a train generated by DLA, thus we can consider the given charge density $\sigma=f \sigma_{36}$ with $N=10$ injection and numerically solve Eq. (9). For comparison, higher value of $N=72$ is also used in our calculation.

Figure (4) shows $\frac{\Delta_{f}}{\Delta_{t}}$ as a function of $f$ for $N=10,36$, and 72 . The results confirm that $\frac{\Delta_{f}}{\Delta_{t}} \cong 1$ and the discrepancy is within $4 \%$ even at $f=1$. Due to the relativistic effect,

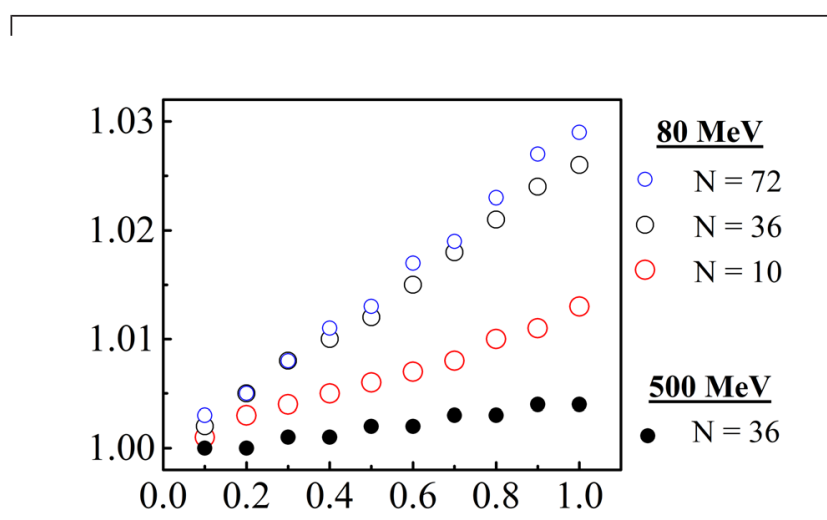

FIG. 4. The dependence of $\Delta_{f}$ (in terms of $\Delta_{t}$ ) as a function of $f$ for $\Delta_{t}=3[\mathrm{fs}$ ] for $N=10,36$ and 72 with $80 \mathrm{MeV}$ injecting energy and gap spacing $d=5 \mathrm{~mm}$ (open circles) and for $N=36$ with $500 \mathrm{MeV}$ injecting energy and gap $\mathrm{d}=31 \mathrm{~mm}$ (solid circles). 
electrons are heavier, and more difficult to be accelerated (or decelerated) when propagating in the drift space. Thus, the high energy train of charge sheet is much easier to maintain the temporal profile and Eq. (4) is able to provide a good estimation because the deviation is less than $4 \%$ (at $f=1$ ). To illustrate that at very high energy, the deviation is less than $1 \%$ around $\frac{\Delta_{f}}{\Delta_{t}}=1$, a case of $500 \mathrm{MeV}$ with same $\Delta T=3$ fs and $N=36$ (but at higher $d=31 \mathrm{~mm}$ ) is plotted (solid black circles) in Fig. 4. In the future, it will be interesting to do a full relativistic particle-in-cell (PIC) simulation to compare with this finding.

To further verify the theory, the practical case reported in Ref. [3] is taken as another test case. For a beam radius (per bunch) of $r_{b}=42[\mu \mathrm{m}]$, we have charge per bunch $Q=1$ [fC], initial kinetic energy $K_{e}=50[\mathrm{keV}]$, number of bunches $N=19$, temporal pulse spacing $\Delta_{t}=2.16[\mathrm{ps}]$ and the gap spacing $d=5[\mathrm{~cm}]$. The corresponding charge density is about $\sigma=\frac{Q}{\pi r_{b}^{2}} \cong 0.45 \sigma_{175}$. This corresponds to $f=0.45$ which gives $14 \%$ discrepancy between $\Delta f$ and $\Delta t$ based on our calculation.

The $14 \%$ discrepancy seems to be acceptable in Ref. [3] since the purpose of the pulse train is to enhance the power of SP radiation, but not to the time-resolved electron microscopy [6], in which the diagnostics is more sensitive to the time structure of the pulse train. Moreover, the main interaction region between the pulse train and the grating for generating SP radiation is near the center of the drift space, thus the temporal separation is relatively wellmaintained as compared to the anode region with higher degradation as shown in Fig. 2(b).

Finally, our theory is a 1D sheet model, therefore the traverse effect is ignored. The traverse effect would decrease the charge density while charges are moving and may help maintain better temporal separations. This fact is supported by Refs. $[27,28]$. We would also like to comment on the validity of the charge sheet model. The electron pulse lengths are $\tau_{p} \cong 20[\mathrm{fs}]$ in Ref. [3] and $\tau_{p} \cong 1$ [fs] in Ref. [2]. Both of the corresponding normalized pulse lengths are $X_{\mathrm{DSCL}} \equiv \frac{\tau_{p}}{T_{\mathrm{DSCL}}} \cong 10^{-5} \ll 1$, which is consistent with the assumption of our model. Prior works by Valfells et al. [17], Zhang et al. [22], and most recently by Liu et al. [24] had also clearly indicated that analytical results based on charge sheet model agree very well with the particle-in-cell or many-body simulation as long as $X_{\mathrm{DSCL}}<0.1$. The Nsheets model also appears in Ref. [29] as a Monte Carlo simulation which has a distinct parallel interest with our works. It is worthwhile to mention it as the end of this paper.

\section{SUMMARY}

In summary, a theoretical model in the paper is constructed to study the space charge effects of $N$ number of charge sheets injected into a drift space with an initial kinetic energy injection up to $80 \mathrm{MeV}$ (including relativistic effect). A formula [Eq. (4)] is derived to determine the upper limit of the charge density per pulse to maintain the uniform time structure of the pulse train. The model may be useful in the design of Smith-Purcell radiation, multiple-pulse electron beam for time resolved electron microscopy, free electron laser or any applications with charge pulse trains over a wide range of parameters.

\section{ACKNOWLEDGMENTS}

The work was supported by National Science Council under the grants MOST (Ministry of Science and Technology) 104-2112-M-013-MY3. Y. L. Liu and S. H. Chen would like to acknowledge the National Center for High-Performance Computing in providing resources under the national project, "Taiwan Knowledge Innovation National Grid," and support by the National Center for Theoretical Sciences, Taiwan, ROC. Y. L. Liu was supported the Singapore MOE T2 grant (T2MOE1401) as a research staff at SUTD. P.Z. was supported by AFOSR Grant No. FA9550-14-1-0309.

[1] H. S. Sim, S. Park, T.-H. Kim, Y. K. Choi, J. S. Lee, and S. H. Lee, Mater. Trans., JIM 51, 1156 (2010).

[2] M.-W. Lin, Y.-L. Liu, S.-H. Chen, and I. Jovanovic, Phys. Plasmas 21, 093109 (2014).

[3] Y. Li and K.-J. Kim, Appl. Phys. Lett. 92, 014101 (2008).

[4] E. Chiadroni, M. P. Anania, M. Artioli, A. Bacci, M. Bellaveglia, A. Cianchi, F. Ciocci, G. Dattoli, D. Di Giovenale, G. Di Pirro, M. Ferrario, G. Gatti, L. Giannessi, A. Mostacci, P. Musumeci, L. Palumbo, A. Petralia, V. Petrillo, R. Pompili, C. Ronsivalle, A. R. Rossi, C. Vaccarezza, and F. Villa, Phys. Procedia 52, 27 (2014).

[5] M. Dashcasan and E. Barati, Phys. Rev. A 89, 043412 (2014).

[6] A. H. Zewail and J. M. Thomas, 4D Electron Microscopy: Imaging in Space and Time, 1st ed. (Imperial College Press, London, Hackensack, NJ, 2009).

[7] S. J. Smith and E. M. Purcell, Phys. Rev. 92, 1069 (1953).

[8] P. Zhang, L. K. Ang, and A. Gover, Phys. Rev. ST Accel. Beams 18, 020702 (2015).

[9] C. D. Child, Phys. Rev. (Series I) 32, 492 (1911).

[10] I. Langmuir, Phys. Rev. 2, 450 (1913).

[11] C. K. Birdsall and W. B. Bridges, Electron Dynamics of Diode Regions (Academic, New York, 1966).

[12] J. W. Luginsland, Y. Y. Lau, and R. M. Gilgenbach, Phys. Rev. Lett. 77, 4668 (1996).

[13] Y. Y. Lau, Phys. Rev. Lett. 87, 278301 (2001).

[14] W. S. Koh, L. K. Ang, and T. J. T. Kwan, Phys. Plasmas 12, 053107 (2005).

[15] W. B. Bridges, J. I. Frey, and C. K. Birdsall, IEEE Trans. Electron Devices 12, 264 (1965).

[16] Y. L. Liu, S. H. Chen, W. S. Koh, and L. K. Ang, Phys. Plasmas 21, 043101 (2014).

[17] Á. Valfells, D. W. Feldman, M. Virgo, P. G. O’Shea, and Y. Y. Lau, Phys. Plasmas 9, 2377 (2002). 
[18] L. K. Ang, T. J. T. Kwan, and Y. Y. Lau, Phys. Rev. Lett. 91, 208303 (2003).

[19] P. Zhang, Sci. Rep. 5, 9826 (2015).

[20] W. S. Koh, L. K. Ang, and T. J. T. Kwan, Phys. Plasmas 13, 063102 (2006).

[21] L. K. Ang and P. Zhang, Phys. Rev. Lett. 98, 164802 (2007).

[22] P. Zhang, W. S. Koh, L. K. Ang, and S. H. Chen, Phys. Plasmas 15, 063105 (2008).

[23] S. H. Chen, L. C. Tai, Y. L. Liu, L. K. Ang, and W. S. Koh, Phys. Plasmas 18, 023105 (2011).

[24] Y. L. Liu, P. Zhang, S.-H. Chen, and L. K. Ang, Phys. Plasmas 22, 084504 (2015).
[25] Y. Zhu and L. K. Ang, Appl. Phys. Lett. 98, 051502 (2011).

[26] Y. Zhu, P. Zhang, A. Valfells, L. Ang, and Y. Lau, Phys. Rev. Lett. 110, 265007 (2013).

[27] A. Pedersen, A. Manolescu, and Á. Valfells, Phys. Rev. Lett. 104, 175002 (2010).

[28] K. Torfason, Á. Valfells, and A. Manolescu, Phys. Plasmas 22, 033109 (2015).

[29] K. L. Jensen, D. A. Shiffer, I. M. Rittersdorf, J. L. Lebowitz, J. R. Harris, Y. Y. Lau, J. J. Petillo, W. Tang, and J. W. Luginsland, J. Appl. Phys. 117, 194902 (2015). 